\title{
Dominic Thomas, Black France. Colonialism, Immigration and Transnationalism
}

\section{Alessandro Corio}

\section{(2) OpenEdition}

1 Journals

\section{Edizione digitale}

URL: http://journals.openedition.org/studifrancesi/9046

DOI: $10.4000 /$ studifrancesi.9046

ISSN: 2421-5856

\section{Editore}

Rosenberg \& Sellier

\section{Edizione cartacea}

Data di pubblicazione: 1 octobre 2008

Paginazione: 493-494

ISSN: 0039-2944

\section{Notizia bibliografica digitale}

Alessandro Corio, «Dominic Thomas, Black France. Colonialism, Immigration and Transnationalism»,

Studi Francesi [Online], 155 (LII | II) | 2008, online dal 30 novembre 2015, consultato il 14 janvier 2021. URL: http://journals.openedition.org/studifrancesi/9046; DOI: https://doi.org/10.4000/studifrancesi. 9046

Questo documento è stato generato automaticamente il 14 janvier 2021.

\section{(c) $($ ) $\odot$ EY}

Studi Francesi è distribuita con Licenza Creative Commons Attribuzione - Non commerciale - Non opere derivate 4.0 Internazionale. 


\title{
Dominic Thomas, Black France. Colonialism, Immigration and Transnationalism
}

\author{
Alessandro Corio
}

\section{NOTIZIA}

DOMINIC THомAS, Black France. Colonialism, Immigration and Transnationalism, Bloomington \& Indianapolis, Indiana University Press, 2007, pp. 305.

1 Il volume si presenta come un interessante e ben riuscito tentativo di applicazione dell'approccio critico, ora egemone nell'ambito dell'accademia americana, ma spesso guardato con sospetto nei dipartimenti di studi letterari in Francia, dei cosidetti cultural studies al contesto letterario francofono. In particolare, lo studio di Dominic Thomas si rivolge, citando implicitamente il titolo dell'ormai celeberrimo The Black Atlantic di Paul Gilroy (Harvard University Press, 1993), ad uno studio approfondito e nutrito di molteplici apporti disciplinari e teorici di quello spazio transnazionale e "transcoloniale", come lui stesso lo definisce nel capitolo introduttivo, della "Black France". Si tratta, dunque, di un oggetto estremamente complesso, dal punto di vista storico-culturale e letterario, che non può essere indagato facendo riferimento semplicemente a concetti, come quello di "diaspora", di "postcolonialismo" o di "letteratura della migrazione" che rischiano di risultare, nonostante l'innegabile valore euristico, omologanti o totalizzanti, non riuscendo a cogliere la pluralità di sfaccettature e le singolarità che emergono dai singoli testi, nonché le tensioni che li attraversano.

2 Per questo, il lavoro di Thomas si nutre di una vasta bibliografia che fa riferimento, oltre ai lavori dei principali critici postcoloniali anglofoni, anche ai fondamentali lavori sull'eredità coloniale della repubblica francese, sull'etnicizzazione del sociale e i rapporti tra immigrazione e cittadinanza, sui rapporti di potere nel campo letterario, 
condotti in ambito "francofono" negli ultimi anni da - per citarne solo alcuni - Achille Mbembe, Etienne Balibar, Jean-Loup Amselle, Pascale Casanova, Odile Cazenave, Pascal Blanchard e Sandrine Lemaire.

3 Il contesto diasporico e transculturale preso in esame permette dunque all'autore di impostare un'analisi critica della tendenza assimilazionista ed integrazionista francese, per quanto riguarda le politiche migratorie e di cittadinanza, e del perdurare di un "discorso coloniale" che informa ancora oggi il pensiero ufficiale sulle questioni dell'immigrazione in Francia. Il libro estende l'analisi dall'epoca coloniale a quella postcoloniale, riconoscendo l'importanza di Parigi come centro di «accumulazione e riproduzione del capitale culturale francofono», ma al tempo stesso "provincializzando" la stessa esperienza migratoria con l'insistere su una dimensione sopranazionale. Come afferma l'autore in un passaggio del primo capitolo, «Black France's claim to originality comes from its attempt to broaden the category of immigrant literature by consulting existing research on minority cultures and diasporic texts, and extending the parameters of research beyond the tradition that has tended to locate immigrant narratives as intrinsically post-Independence or postcolonial phenomena» (p. 38).

4 Così, nel secondo capitolo i testi letterari pubblicati prima delle indipendenze da autori africani, come Mirage de Paris (1937) di Ousmane Socé, L'Enfant noir (1954) di Camara Laye, Un nègre à Paris (1959) di Bernard Dadié, Chemin d'Europe (1960) di Ferdinand Oyono e L'aventure ambiguë (1961) di Cheikh Hamidou Kane, vengono considerati come "narrative migranti", per il fatto che affrontano i temi del dislocamento socioeconomico e culturale tra l'Africa e la métropole. L'analisi della blackness è poi ampliata, nel capitolo successivo, ad un livello "globale", con una lettura comparata di Le docker noir (1956) di Ousmane Sembene e Native Son (1940) di Richard Wright. Il quarto capitolo si situa, invece, strategicamente lungo la giuntura tra l'epoca coloniale e quella postcoloniale, affrontando il tema della schiavitù nelle sue forme contemporanee attraverso l'analisi di un racconto di Ousmane Sembene del 1962, La noire de... e della narrazione autobiografica contemporanea di Henriette Akofa, Une esclave moderne (2000). Il quarto e quinto capitolo mettono al centro criticamente la questione della ricezione, sottolineando in che modo alcuni testi di forte valore testimoniale possano essere paradossalmente recuperati, entrando nel mercato editoriale, dalle "autorità culturali esagonali" in senso culturalista e, dunque, come strumenti di esclusione. Al centro del quinto capitolo è la controversa e dibattuta questione dell'escissione, sia nell'Africa francofona subsahariana che nel contesto della diaspora africana in Francia, attraverso la lettura del romanzo di Fatou Keïta, Rebelle (1998), e di due "lettere" di Calixthe Beyala: Lettre d'une africaine à ses sœurs occidentales (1995) e Lettre d'une Afro-française à ses compatriotes (2000). La tematica sociale, culturale e morale dell'escissione è così affrontata in una dimensione transculturale, tenendo presenti gli scambi discorsivi che avvengono tra le differenti location della diaspora.

Il sesto capitolo prende poi in considerazione un altro significativo fenomeno transculturale, quello dei sapeurs, in cui i codici estetici europei vengono riposizionati nello spazio della diaspora congolese, attraversando questioni di genere e di performance sociale e soggettiva nella marginalità. Il fatto sociale viene letto attraverso il romanzo Bleu, Blanc, Rouge (1998) di Alain Mabanckou e i due romanzi di Daniel Biyaoula, L'impasse (1996) e Agonies (1998), dove la Francia e Parigi assumono un valore di spazio mitico per l'acquisizione di "capitale simbolico" nella diaspora africana. 
Infine, l'ultimo capitolo considera altri sviluppi del fenomeno migratorio nell'ambito di un'economia globale, simbolica e materiale, nel romanzo del 2003 di Fatou Diome, Le ventre de l'Atlantique, che lancia una sfida innovativa al mito della migrazione discusso in precedenza. Il romanzo della Diome consente, così, di mappare alcune nuove coordinate di una geometria transnazionale della diaspora, in cui la periferia viene riposizionata costantemente come un altro centro, capace di rimettere in discussione le politiche di sviluppo, gli aggiustamenti strutturali e l'impatto della globalizzazione, rivolti unidirezionalmente verso il Sud. 